\title{
A Small-Scale Study of the Effects of Supplemental Vocabulary Instruction on Preschoolers with Vocabulary Delays
}

\author{
Kathleen Roskos $^{1 *}$, Karen Burstein ${ }^{2}$, Shannon Sullivan ${ }^{1}$ \\ ${ }^{1}$ John Carroll University, University Heights, USA \\ ${ }^{2}$ Southwest Institute for Families and Children, Scottsdale, USA \\ Email: *roskos@jcu.edu
}

Received May $7^{\text {th }}$, 2013; revised June $7^{\text {th }}$, 2013; accepted June $14^{\text {th }}$, 2013

Copyright (c) 2013 Kathleen Roskos et al. This is an open access article distributed under the Creative Commons Attribution License, which permits unrestricted use, distribution, and reproduction in any medium, provided the original work is properly cited.

\begin{abstract}
Research on preschool vocabulary instruction has increased considerably as the need for stronger early literacy programs has garnered public attention. While research findings show moderately to large effects of direct, intensive vocabulary instruction on children's word learning, results are less robust for those children with vocabulary delays. In general, design specifications of more effective interventions remain unclear. Using a matched sample design, this study examined whether greater frequency of a direct, intensive vocabulary intervention alone improved gains for children with vocabulary delays, or if a more complex treatment may be needed. Participants included 24 children with vocabulary delays were drawn from eight Head Start classrooms in Early Reading First programs. Results of the study indicated that increasing the frequency of an intensive intervention yielded notable gains for children resistant to vocabulary instruction. Implications for early literacy instructional practice are discussed.
\end{abstract}

Keywords: Preschool Vocabulary Instruction; Word Learning Intervention; Vocabulary Delays; Intervention Design

\section{Introduction}

Research on preschool vocabulary instruction has increased considerably in the last decade stimulated by the surge of educational attention on early literacy development and achievement (e.g., NELP, 2008). Based on evidence that the volume of word learning in the early years has a profound impact on future reading comprehension (Hart \& Risley, 2003), studies have focused on teaching practices and interventions that support vocabulary development in young children, especially those with vocabulary delays. In general, this research shows the benefits of direct and intensified vocabulary instruction in promoting vocabulary development and growth, although specific features of implementation vary (e.g., word selection) (Silverman \& Crandall, 2010). It also consistently shows that children with stronger vocabularies at the outset gain more from instruction than those with weaker vocabularies (Ehri, 2005). Studies, however, also consistently show that those children with weak vocabularies make gains, but not enough to overcome the drag of delay on their progress (Margulis \& Neuman, 2009). How to intervene and increase these children's modest vocabulary gains more substantively remains an open question. It's not clear if heavier doses of existing approaches or new, specially designed interventions are needed to address the problem. In this study we investigate whether more of the same can improve the vocabulary gains of preschoolers with delays, testing the design strength of an instructional supplement used in prior research.

\footnotetext{
*Corresponding author.
}

\section{Background of the Study}

The weak response to intervention for those children with vocabulary delays has prompted researchers to design more direct and intensive interventions that might stimulate change in word volume and rate. Neuman, Newman \& Dwyer (2010), for example, developed a taxonomic approach to word learning, referred to as World of Words or WOW, grounded in the evidence of a "tight" correlation between the emergence of word categories (taxonomic structures) and an increase in word learning (Borovosky \& Elman, 2006). The efficacy of the WOW technique is promising showing significant gains for the treatment group in word knowledge and concept development; however, the stability of the intervention is unclear since it appears to work better with some content topics over others. It was less effective, for instance, on topics with more abstract mathematical concepts and words.

Exploiting the appeal (and explosion) of technology, other researchers have examined the potential of educational software and electronic books for boosting word learning in children with language delays. In a series of studies, Segers \& Verhoeven $(2002,2003,2008)$ tested the potential of Treasure Chest with the Mouse (TCM), an interactive vocabulary book, to improve the literacy skills of kindergarteners, namely print awareness, vocabulary and sound-symbol matching. Significant effects for word learning were found among children participating in TCM as compared to a control, although no effect showed up on a standardized vocabulary test. While this may be accounted for as resistance to treatment noted in other studies, it also raises the possibility that conventional measures may 
lack sensitivity and specificity to detect subtle changes in vocabulary development.

Focused on e-book media, Verhallen and colleagues (Verhallen, Bus, \& de Jong, 2006) argue that digitized storybooks with film-like visualizations, audio and other additions support language/literacy development in two powerful ways: 1) added images, sounds and emotions facilitate oral language comprehension and memorization and 2) onscreen books mobilize energy so children invest more mental effort in oral language comprehension. Their studies show that children lagging in language skills benefit most, learning $2 \times$ as many words in multiple electronic readings over encounters with static books (Verhallen, Bus, \& de Jong, 2006). These children are also more active in multi-media ebooks, demonstrating higher rates of skin conductance responses indicative of increased amounts of invested mental effort toward story comprehension (Verhallen \& Bus, 2009). Still, the modest effect size $\left(\eta_{p}^{2}=.16\right)$ in this study underscores the stubborn resistance to intervention among children with weak language skills.

"Engineering" the traditional shared book reading procedure to foreground word learning, several researchers have developed more deliberate instructional sequences that attempt to teach a target set of words. Silverman (2007), for example, describes Anchored Vocabulary Instruction that combines oral contextual strategies (e.g., linking new words to personal experience) and analytical strategies (e.g., attending to letters and sounds of new words) that bridge oral and print sources of word meanings. Anchored vocabulary instruction had a large effect size on the sample $\left(\eta_{p}^{2}=.94\right)$, and researchers found this type of vocabulary instruction to be more effective than contextual or analytical instruction alone. More direct, Biemiller and Boote (2006) tested the effects of well-placed vocabulary interruptions to explain word meanings in a repeated readings approach with positive results, especially for kindergarteners. The effect size for overall pre- and post-test scores was large (Cohen's $d=1.21$ ) and that the main effect was highly significant $(F=182.73)$. Similarly, Smeets and Bus (2012) found that multiple-choice questions embedded in an interactive electronic storybook either during or after reading increased word learning a sizeable percentage over a read only condition. Moreover this approach outperformed the hotspot as a source of vocabulary instruction. Direct, intensive, embedded vocabulary instruction, it appears, may substantially reduce resistance to intervention in children with vocabulary delays.

Several instructional features emerge from these different approaches that may be viewed as design basics of vocabulary intervention: 1) a before-during-after (BDA) instructional framework to introduce, discuss and review new words (format); 2) repetition and explanation of new words in context (instruction); and 3) opportunities to use new words in contexts beyond the book (transfer). Specifications of intensity, frequency, duration and conditions of instructional approaches incorporating these design basics, however, remain unclear. Questions, such as how strong; how often; for how long; and in what ways in the classroom setting supplemental vocabulary instruction should occur require further research. In the final analysis, it is probably not one instructional approach over another (a magic pill), but rather its prescription, or set of instructions, for providing secondary prevention of vocabulary delay that matters.

In this study we focus on the impact of instructional frequency on children's word learning gains when provided an instructional supplement, referred to as say-tell-do-play (STDP).
Research on the frequency of vocabulary instruction in early childhood classrooms is rare. A recent study conducted in kindergarten classrooms reports an average of 8.14 discussions (not instruction) of vocabulary words per day that are very brief, episodic and limited to relatively easy words (Wright, 2012). The STDP supplement replicated a direct, intense vocabulary instruction approach routinely used in the preschool literacy curriculum to teach sets of target words (8 - 10 per set). Our research goal was to examine the impact of instructional frequency on 1) overall receptive vocabulary growth of those receiving the supplemental instruction and 2) the number of target words learned over a treatment period. We hypothesized that more of the same (direct, intense instruction) over a treatment period yields greater word learning gains for children vulnerable to vocabulary delay. Rate of word learning during treatment and fidelity of implementation (accuracy) were also examined.

\section{Method}

We used a matched subject's research design to test our general hypothesis that an increase in the frequency of direct, intense vocabulary instruction boosts its efficacy for vulnerable children. If demonstrated, this factor has implications for using the STDP technique in early childhood classrooms and informs the use of similar techniques aimed at secondary prevention.

\section{Setting}

The study was conducted in eight Head Start classrooms in their third year of an Early Reading First program. The program emphasized the development of essential early literacy skills using scientifically research-based techniques and strategies (Whitehurst, 2008). Classrooms were located in the Midwest (n $=4)$ and the Southwest $(n=4)$ United States. The teacher-child ratio in each of these classrooms was on average 1:10. Each classroom included a teacher assistant.

The literacy curriculum represented an evidence-based approach per Early Reading First guidelines (e.g., provide activeties and instructional materials based on scientifically based reading research for use in developing children's phonological awareness (ERF Guidance, p. 9)). The curriculum was embedded in a series of content topic studies (e.g., Buildings) that are part of the Creative Curriculum (Dodge, Colker, \& Heroman, 2002). Children received on average a total of 75 minutes of daily early literacy instruction divided between whole group shared book reading and small group literacy activities at activity centers. All teachers used a direct, intense vocabulary instruction technique in the context of shared book reading, referred to as say-tell-do-play (See instructional example in Appendix). Target words taught in the context of shared book reading followed a say-tell-do technique and were further practiced in play activities in activity centers on a daily basis.

\section{Participants}

From the 8 classrooms, children with a standard score of 85 or less on the PPVT-IV (Dunn \& Dunn, 2007) were identified as vocabulary-delayed, totaling 161 children. From this group of eligible children, 24 children were randomly selected for participation in the study. From this eligible pool, a sub-sample of 12 children was assigned to treatment, referred to as eligible treatment (ET; 9 males; 3 females; mean age $=54.3$ months; 
mean PPVT standard score $=79$ ). A matched sample was assigned to a non-treatment condition, referred to as eligible nontreatment (EN; 9 males; 3 females; mean age = 53.1 months; mean PPVT standard score $=79$ ). A total of 10 teachers participated, holding associate or BS degrees and averaging 22 years of experience. All had received an estimated 10 hours of training in the STDP technique as a part of ongoing professional development in the Early Reading First program.

\section{STPD Supplement}

The STDP supplement replicates the say-tell-do-play vocabulary approach embedded in the daily early literacy instruction of the participating classrooms. The instructional design of STDP is grounded in the basic research on the statistical and social nature of vocabulary development. From infancy the amount and type of language available in the environment shapes vocabulary development (Samuelson, 2002 [IES]). Studies of infant speech, for example, show that sound frequencies in the environment shape the neural architecture for language (RiveraGaxiola, Silva-Pereya, \& Kuhl, 2005). At the same time, vocabulary acquisition is social, requiring human interaction for word learning to occur (Hockema \& Smith, 2009; Kuhn, Tsao, \& Liu, 2003). As Hart and Risley (2003) demonstrated, children grow more like their parents in vocabulary resources, language and interaction style. By age 3 they talk and use numbers of different words similar to averages of their parents. The social setting creates the environmental conditions for fast mapping that links sound information in the environment to meaning in individual working memory, and sets the stage for schema construction in long-term memory (Bloom, 2002; Carey, 1978; Heibeck \& Markman, 1987; Smith, 2000).

Based on this body of research, two instructional design features appear critical for directly teaching vocabulary to young children: 1) multiple exposures to new words and 2) social interaction in the word-learning environment. The instructional design goal is to support meaningful word learning that engages the young learner in substantial cognitive processing yet is sensitive to cognitive load, i.e., does not overwhelm working memory (Sweller, van Merrienboer, \& Paas, 1998). Achieving this goal rests on three assumptions of cognitive processing (Mayer \& Moreno (2003: p. 44) — the dual channel assumption (separate information processing channels for verbal and visual material) (Paivio, 1986); the limited capacity assumption (limited amount of processing capacity available in dual channels) (Chandler \& Sweller, 1991); and the active processing assumption (learning requires cognitive work in each channel) (Wittrock, 1981).

Illustrated in Figure 1, the instructional design of STDP includes repetitive tasks across three phases of word exposure in a storybook context that involve auditory-visual processing channels: looking at and saying the word (print + picture); telling about the word; and using a gesture for the word. Each task is designed to help young learners pay attention to target words, organize them and integrate them within the context of an unfolding story as well as their own existing knowledge. The task sequence concludes with a play activity based on play research that shows the strong social "press" for meaningful language use in play contexts (Pellegrini \& Galda, 1998; Nicolopoulou, 2005). Moreover the opportunity for play maintains motivation to learn new words because it is fun and enjoyable for children.

So designed, STDP provides maximum exposure to new words in a storybook context through auditory and visual channels (Paivio, 1990) in close succession so as to reduce cognitive load (Sweller, van Merrienboer, \& Paas, 1998). It offers children a learning strategy for saying, telling and "doing" words before, during and after reading followed by play activity, which encourages personal language use (Pellegrini \& Galda, 1993).

Two times a week over a 12-week period, teachers implemented the STDP supplement to the ET group in a quiet setting following the steps outlined in Table 1. Prior to reading, children were introduced to 3 - 5 target words from the story aligned with the current topic study. Word cards with photos and/or concrete objects were used to present each word. Following modeling by the teacher, each child was encouraged to say, tell about and enact a gesture (do) in association with the word. During reading, the teacher highlighted or repeated the

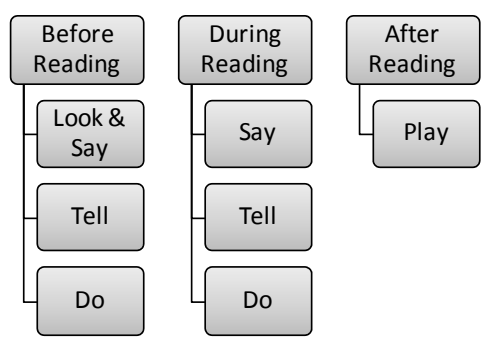

Figure 1.

STDP design.

Table 1.

STDP implementation protocol.

\begin{tabular}{|c|c|c|c|}
\hline Read aloud: & Date: & & \\
\hline \multirow[t]{2}{*}{ Target words: } & Photos: & Yes & No \\
\hline & Objects: & Yes & No \\
\hline \multirow[t]{3}{*}{ Phase } & Step & Protocol & Teaching actions \\
\hline & 1 & I say & $\begin{array}{l}\text { T says target word } \\
\text { w/photo or object }\end{array}$ \\
\hline & 2 & You say & $\begin{array}{l}\text { T asks children to } \\
\text { say target word }\end{array}$ \\
\hline \multirow[t]{5}{*}{ Before reading } & 3 & I tell & $\begin{array}{l}\mathrm{T} \text { tells meaning of target } \\
\text { word w/photo or object }\end{array}$ \\
\hline & 4 & You tell & $\begin{array}{l}\text { T asks children to } \\
\text { turn- } n \text {-tell a friend }\end{array}$ \\
\hline & 5 & \multicolumn{2}{|c|}{${ }^{*}$ Repeat for each word } \\
\hline & 6 & I say \& tell & T says \& tells target words \\
\hline & 7 & You say \& tell & $\begin{array}{l}\text { T asks children to } \\
\text { say \& turn-n-tell }\end{array}$ \\
\hline \multirow[t]{3}{*}{ During reading } & 8 & I do & $\begin{array}{l}\text { T uses action to help } \\
\text { define new word }\end{array}$ \\
\hline & 9 & You do & $\begin{array}{l}\text { T asks children to } \\
\text { repeat action }\end{array}$ \\
\hline & 10 & Let’s play & $\mathrm{T}$ invites children to play \\
\hline After reading & & & $\begin{array}{l}\text { T encourages use of target } \\
\text { words in play }\end{array}$ \\
\hline
\end{tabular}


words, inviting children to say-tell-do specific words in the context of the story. After reading, she briefly reviewed the words and then moved into a simple play activity (memory game; board game; puppet play) where children were encouraged to use new words on their own. The supplement, therefore, replicated the instruction used in the general curriculum, providing an extra dose of direct, intense vocabulary instruction.

\section{Measures}

All children in the ERF program were administered the PPVT-IV as pre/post measure of receptive vocabulary. A curriculum-based measure, referred to as LearningWell CBDM (Burstein; http://cbdm.defaultroute.net/) was used to monitor the word learning of children in the ET and EN groups on a weekly basis during the treatment period. To measure receptive vocabulary, individual children were shown a screen with a photo set, including the topic-related target words and two foil words. They were asked to point to the target words (Show Me). To measure expressive vocabulary, the child was shown a single photograph and asked to name the picture or action in the picture (Tell Me). In both groups the assessment was administered at the end of each week on words taught that week.

The 10-step instructional protocol (Table 1) was used to guide/support fidelity of implementation. Using video samples, the research team monitored implementation by coaches, providing feedback in one-to-one exchanges and in professional development. Over the 8-week period, coaches monitored teachers using direct observation based on the 10-step protocol; they provided feedback on fidelity on-the-spot and in weekly professional development sessions.

\section{Procedures}

Following the assignment of eligible children to either treatment or non-treatment groups, the STDP supplement was implemented in 8 classrooms over a 12-week period. Initially literacy coaches implemented STDP two times a week for an estimated 4 weeks with the children to establish routines and model the protocol with a small group. Next teachers implemented the procedure twice weekly for 8 weeks. All total, the ET group received about 30 minutes of supplemental vocabulary instruction on 3 - 5 topic-related target words each week. This was in addition to the estimated 60 minutes they received in daily instruction on 8 - 10 topic-related words per week. The EN group received daily vocabulary instruction on the $8-10$ topic-related target words only (See Table 2).

\section{Data Analysis}

Pre/post scores on the PPVT-IV were compared as a direct

Table 2.

Instructional time and focus in general and supplemental vocabulary instruction.

\begin{tabular}{cccccc}
\hline Instruction & General & $\begin{array}{c}\text { Total } \\
12 \text { weeks }\end{array}$ & Supplemental & $\begin{array}{c}\text { Total 12 } \\
\text { weeks }\end{array}$ & $\begin{array}{c}\text { Total } \\
\text { ET }\end{array}$ \\
\hline & EN and ET & \multicolumn{3}{c}{ ET only } \\
\hline $\begin{array}{c}\text { Minutes per } \\
\text { week }\end{array}$ & $\sim 1$ hour & $\sim 12$ hours & $\sim 30$ minutes & $\sim 6$ hours & $\sim 18$ hrs \\
$\begin{array}{c}\text { Words per } \\
\text { week }\end{array}$ & $\sim 10$ & $\begin{array}{c}\sim 120 \\
\text { words }\end{array}$ & $\sim 5$ & $\sim 60$ words & $\begin{array}{c}\sim 180 \\
\text { words }\end{array}$ \\
\hline
\end{tabular}

measure of children's receptive vocabulary growth. Since the sample size was small ( $\mathrm{n}=20$; 4 children left the program because their parents re-located [3 from EN; 1 from ET]), statistical tests were not conducted. A scatter plot was used to visually examine correlational trends between groups in a preliminary way. Weekly progress monitoring data were analyzed using descriptive statistics. Overall mean gains between groups on the PPVT and CBDM tasks were calculated as percentages. Rate was defined as the number of target words identified per session and was calculated for the ET group only. Performance scores on the assessment tasks were rated as high (majority of words identified; rating $=3$ ); average (half of total words identified; rating = 2); and low (1 or 0 words identified; rating =1).

Using the 10-step STDP implementation protocol (Table 1), the research team $(n=3)$ selected a 10-minute video sample of each coach's STDP instruction (the equivalent of one session) to check fidelity to the protocol. Each step was coded as present (1) or not present (0) on a majority of target words (>50\%) for items 1-9 and the play activity. Inter-observer reliability was established at nearly $100 \%$ on a coach sample.

Coaches were assigned 2 or 3 teachers and used the protocol to observe each teacher's STDP implementation 4 times staggered over the 8 -week period (equivalent to $25 \%$ of sessions). Inter-observer reliability was established at $92 \%$ on a randomly drawn sample of three observations from a pool of 40 observations. Observations of fidelity of implementation on the 10-step protocol for each teacher were summed ( $\mathrm{n}=4$ protocols); an individual average of $80 \%$ of steps present was considered adequate.

\section{Results}

\section{Fidelity of Implementation}

Fidelity of implementation was highest among coaches with a mean of $90 \%$ per the protocol and a range of $80 \%-100 \%$. Teachers implemented the protocol with a moderate degree of fidelity with a mean of $80 \%$ and a range of $70 \%$ - 90\%. Differences occurred primarily around the number of target words subjected to the say-tell-do routine in an instructional session, at times failing to reach the $>50 \%$ of words criterion. This, in turn, impacted the overall strength of the instructional supplement in the session. The play portion of the protocol was implemented consistently (and joyfully) across educational staff and sessions.

\section{Receptive Vocabulary Growth in ET and EN Group}

Figure 2 compares the pre/post performance on the PPVTIV of the ET and EN matched groups. These results show that the ET group gained nearly 13 percentage points in receptive vocabulary knowledge whereas the EN group gained approximately 7 points, giving the ET group about a 6-point advantage. That the majority of the children in the ET group $(n=10)$ had a post PPVT-IV standard score above 85 is also notable, suggesting that they may no longer need supplemental instruction beyond general classroom instruction, although further research is needed to test the sustainability of the intervention. Additionally, the insensitivity of standardized measures, such as the PPVT, to yearly vocabulary growth should be considered when gauging the practical impact of the intervention.

While the sample is very small, the scatter plot in Figure 3 provides a preliminary view of the relative strength of the rela- 
tionships between treatment/non-treatment and vocabulary gains for each group. As the trend lines show, there is a positive correlation between the vocabulary instruction and word learning for both groups, although the correlations are in the low range $(\mathrm{EN}=.139$; $\mathrm{ET}=.423)$; this is no surprise given the resistance to treatment often found in vocabulary-delayed children. The difference in the linear values between the groups, however, is noteworthy indicating that the treatment (more of the same STDP) accounted for about $30 \%$ more of the variance in vocabulary gain for the ET group over the EN group. In other words, increased frequency of STDP increased its power to impact word learning about three-fold for these children. What this suggests is that more frequent use of an intense supplement alone may modestly improve children's chances of word learning. Statistical tests with a larger sample are needed to observe if such increases hold or even improve significantly.

\section{Target Word Learning in ET and EN Groups}

Figure 4 shows the overall mean comparison of percentage of target words identified between ET and EN groups on the progress monitoring tasks of Show Me and Tell Me.

Eligible children receiving the STDP instructional supplemental demonstrated an 8-point advantage over their peers in their

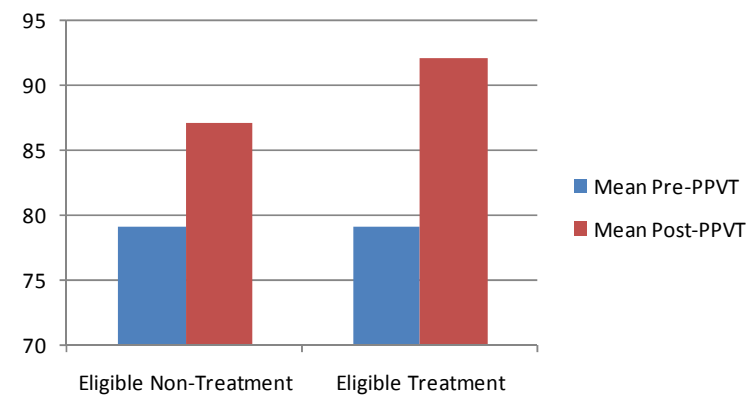

Figure 2.

Comparison of receptive vocabulary standard scores between groups.

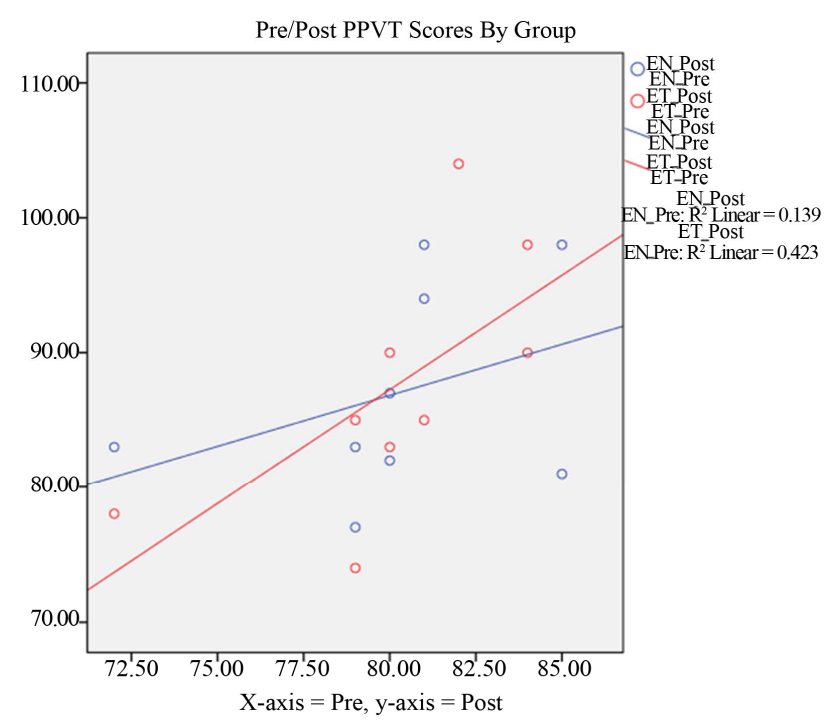

Figure 3.

Scatter plot of EN and ET pre/post PPVT standard scores. learning of receptive vocabulary (Show Me) and 9 points in their expressive vocabulary (Tell Me). These are substantial differences, and need to be tested for significance with a larger sample.

\section{Description of Rate of Word Learning}

The analysis of word learning rate in the ET group is summarized in Figure 5. In general, the majority of children in the ET group demonstrated an average rate of word learning in both receptive and expressive modes. In short, they identified and verbalized about half the words after a week of instruction. That the word-learning rate in the expressive mode for the majority of children is in the average range is noteworthy. Half of the children showed a high rate of word learning in the recaptive mode, identifying most or all of the target words; however, none performed at a high rate in the expressive mode. Two of the children showed a low rate of word learning in their expressive vocabulary.

\section{Discussion}

How to improve children's word learning via direct instruction remains elusive, although recent studies have foregrounded what appear to be potent features of intervention (e.g., anchored instruction). The various combinations of intensity, frequency, duration and conditions of instruction matched to individual needs will require considerably more research—as there is unlikely any universal treatment, especially for children with serious vocabulary delays. Our study shows that on a small scale, children who received more frequent direct, intensive vocabulary instruction learned more words each week than their peers with less frequent instruction. The majority of children showed an average rate of receptive word learning with most demonstrating average rates. The rate of expressive word learn-

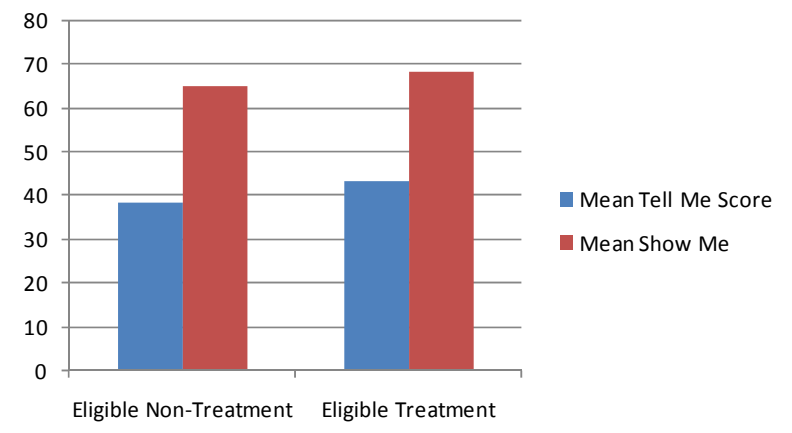

Figure 4.

Percentage of target words identified in CBM.

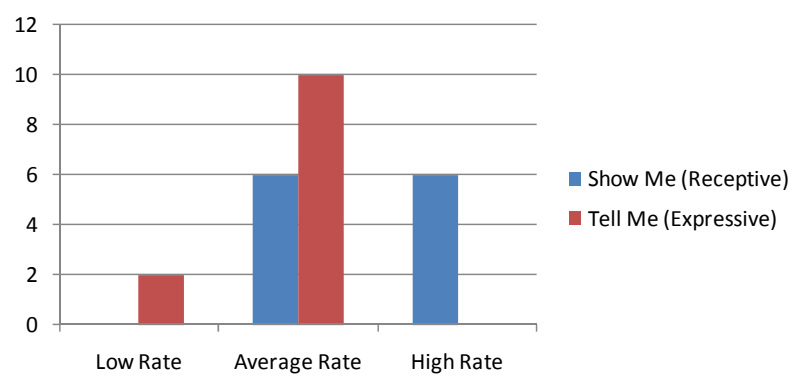

Figure 5.

Rate of word learning in ET group. 
ing, however, remained stubbornly low, also reported in prior research. The findings are preliminary, but warrant further experimental testing of the STDP instructional supplement with a larger sample.

Several design features of the STDP instructional supplement show promise for vocabulary intervention in classroom settings. One is the say-tell-do set of mental strategies that may increase opportunities for word learning multi-modally (looking, speaking, listening, gesturing). Both theory (Paivio, 1990) and research (e.g., Korat, 2010) show the benefits of multi-channel word processing for word learning. That the instruction asks children to say a word, talk about it and attach a gesture to it actively engages them and may help to focus their attention and concretize word meaning.

Another is the close temporal succession of word exposures in a story context, which may help to organize and focus the mental energy needed for word learning. Based on cognitive load theory (Sweller, van Merrienboer, \& Paas, 1998), this makes sense in that the instructional sequence may support ongoing attention to words and word-consciousness. Research on cross-modal attention also shows the benefits of temporal contiguity for focusing attention on word meaning in context (Verhallen \& Bus, 2012).

A third design feature is the opportunity for brief instructional episodes during shared reading that teach and reinforce new words-a feature also supported in related research (Biemiller \& Boote, 2006; Smeets \& Bus, 2012). Some studies, for instance, show that these instructional 'moments' are not disruptive, but rather help children to concretize unknown words and to make connections between story line, pictures and word meanings (Bus \& Verhallen, 2009).

A fourth feature is the inclusion of play activity that may motivate young children to explore and use new words spontaneously, thus boosting their own self-agency with language. Studies of play talk indicate that children tend to use more expressive language (Fein, 1979) and complex syntax (Vedeler, 1997) in play episodes

Our findings also inform the practical application of other direct, intense vocabulary approaches where more of the same may improve efficacious without the need to seriously modify the approach or abandon it altogether. The frequency factor in treatment has advantages in that it is easier to implement in real settings than changes in intensity, which may complicate instruction, and it can influence duration and conditions. The length of treatment, for example, may be reduced; conditions may be met through computer-based instruction.

In sum, the findings of our study indicate that increasing the frequency of the STDP supplement improved its viability with children resistant to vocabulary intervention in the classroom setting. The findings also support its instructional design, which appears to support word learning readiness and effort. Finally, the findings point to the potential of frequency as a key factor in specifying the implementation of robust vocabulary instruction approaches with children at-risk for vocabulary delay.

\section{Limitations}

While the small-scale nature of the study allowed close examination of a matched sample, it also met with several limitations. The classroom settings used in the study were implementing an Early Reading First program that probably favored the STDP instructional approach. Early Reading First (ERF) programs focus on science-based early literacy instruction in well-resourced educational settings (e.g., substantial teacher professional development) that put them at an advantage over non-ERF programs. The matched sample was quite small after attrition, and attendance was not accounted for in the analysis. In combination these factors seriously limit the results, although the results appear compelling enough to warrant further research. Fidelity of implementation indicated potentially significant variability between educational roles (coach; teacher) and between individual teachers. A threshold for implementing the instructional supplement needs to be established to better gauge effects on children's word learning. Inevitable problems with logistics (e.g., schedules; special events; student/staff absences) also compromised the STDP supplement, and may have impacted the results.

\section{Conclusion}

A strong vocabulary is at the core of strong language comprehension, thus accounting for its high priority in preschool language and literacy programs. For children with vocabulary delays, however, general instruction is often insufficient for adequate progress. Supplemental instructional procedures are needed to afford these children more opportunity to learn words and practice using them. Yet the specifics of intensity, frequency, duration and conditions of supplemental instruction as treatment factors are only emerging. As a supplemental approach, greater frequency of the say-tell-do-play procedure during the preschool day shows promise for boosting the word learning of vocabulary-delayed youngsters in a practical way. When intense interventions are in place, frequency may be a viable factor for boosting response to treatment.

\section{REFERENCES}

Biemiller, A., \& Boote, C. (2006). An effective method for building meaning vocabulary in primary grades. Journal of Educational Psychology, 98, 44-62. doi:10.1037/0022-0663.98.1.44

Bloom, P. (2002). Mindreading, communication, and the learning of names for things. Mind and Language, 17, 37-54. doi:10.1111/1468-0017.00188

Borovosky, A., \& Elman, J. (2006). Language input and semantic categories: A relation between cognition and early word learning. Journal of Child Language, 33, 759-790. doi:10.1017/S0305000906007574

Bus, A., \& Smeets, D. J. H. (2012). Interactive electronic storybooks for kindergartners to promote vocabulary growth. Journal of Experimental Child Psychology, 112, 36-55.

Bus, A., \& Verhallen, M. J. A. J. (2009). Video storybook reading as a remedy for vocabulary deficits. Journal for Educational Research Online, 1, 172-196.

Carey, S. (1978). The child as word learner. Linguistic theory and psychological reality In M. Halle, J. Bresnan, \& G. A. Miller (Eds.), Linguistic theory and psychological reality (pp. 264-293). Cambridge, MA: MIT Press.

Chandler, P., \& Sweller, J. (1991). Cognitive load theory and the format of instruction. Cognition and Instruction, 8, 293. doi:10.1207/s1532690xci0804_2

Dodge, D. T., Colker, L. J., \& Heroman, C. (2005). The creative curriculum for preschool (4th ed.). Bethesda, MD: Teaching Stategies Inc.

Dunn, L. M., \& Dunn, L. M. (2007). Peabody picture vocabulary test (4th ed.). Minneapolis, MN: Pearson Assessments.

Ehri, L. C. (2005). Learning to read words: Theory, findings, and issues. Scientific Studies of Reading, 9, 167-188. doi:10.1207/s1532799xssr0902 4 
Fein, G. (1979). Play in the acquisition of symbols. In L. Katz (Ed.), Current topics in early childhood education (pp. 195-225). Norwood, NJ: Ablex.

Hart, B., \& Risley, T. R. (2003). The early catastrophe: The 30 million word gap. American Educator, 27, 4-9.

Heibeck, T. R., \& Markman, E. M. (1987). Word learning in children: An examination of fast mapping. Child Development, 58, 1021-1034. doi:10.2307/1130543

Hockema, S., \& Smith, L. B. (2009). Learning your language, outsidein and inside-out. Linguistics, 47, 453-479. doi:10.1515/LING.2009.016

Korat, O. (2010). Reading electronic books as a support for vocabulary, story comprehension and word reading in kindergarten and first grade. Computers \& Education, 55, 24-31.

doi:10.1016/j.compedu.2009.11.014

Kuhl, P. K., Tsao, F. M., \& Liu, F. M. (2003). Foreign-language experience in infancy: Effects of short-term exposure and social interaction on phonetic learning. Proceedings of the National Academy of Sciences of the United States of America, 100, 9096-9012. doi:10.1073/pnas.1532872100

Margulis, L., \& Neuman, S. B. (2009). The effects of vocabulary intervention on young children's word learning. Paper Presented at the National Reading Conference, Albuquerque.

Mayer, R. E., \& Moreno, R. (2003). Nine ways to reduce cognitive load in multimedia learning. Educational Psychologist, 38, 43-52. doi:10.1207/S15326985EP3801_6

National Early Literacy Panel (2008). Developing early literacy: A scientific synthesis of early literacy development and implications for intervention. Washington DC: US Department of Education. http://lincs.ed.gov/publications/pdf/NELPReport09.pdf

Neuman, S., Newman, E., \& Dwyer, J. (2010). Educational effects of a vocabulary intervention on preschoolers' word knowledge and conceptual development: A cluster-randomized trial. Reading Research Quarterly, 46, 249-272.

Nicolopoulou, A. (2005). Play and narrative in the process of development: Commonalities, differences, and interrelations. Cognitive Development, 20, 495-502. doi:10.1016/j.cogdev.2005.09.001

Paivio, A. (1990). Mental representations: A dual coding approach. Oxford: Oxford University Press.

doi:10.1093/acprof:oso/9780195066661.001.0001

Pellegrini, A. D., \& Galda, L. (1993). Ten years after: A reexamination of symbolic play and literacy research. Reading Research Quarterly, 28, 162175. doi: $10.2307 / 747887$

Pellegrini, A. D., Galda, L., Bartini, M., \& Charak, D. (1998). Oral language and literacy learning in context: The role of social relationships. Merrill-Palmer Quarterly, 44, 38.

Rivera-Gaxiola, M., Silva-Pereyra, J., \& Kuhl, P. (2005). An eventrelated brain potential study of sentence comprehension in pre- schoolers: Semantic and morphosyntactic processing. Cognitive Brain Research, 23, 247-258.

Samuelson, L. K. (2002). Statistical regularities in vocabulary guide language acquisition in connectionist models and 15 - 20-month-olds. Developmental Psychology, 38, 1016-1037.

doi:10.1037/0012-1649.38.6.1016

Segers, E., \& Verhoeven, L. (2002). Multimedia support of early literacy learning. Computers \& Education, 39, 207-222. doi:10.1016/S0360-1315(02)00034-9

Segers, E., \& Verhoeven, L. (2003). Effects of vocabulary training by computer in kindergarten. Journal of Computer Assisted Learning, 19, 557-567. doi:10.1046/j.0266-4909.2003.00058.x

Segers, E., Verhoeven, L., \& Nanneke, H. H. (2008) Cognitive processes in children's multimedia text learning. Applied Cognitive Psychology, 22, 375-338. doi:10.1002/acp.1413

Silverman, R. (2007). A comparison of three methods of vocabulary instruction during read-alouds in kindergarten. The Elementary School Journal, 108, 97-113. doi:10.1086/525549

Silverman, R., \& Crandell, J. D. (2010). Vocabulary practices in prekindergarten and kindergarten classrooms. Reading Research Quarterly, 45, 318-340. doi:10.1598/RRQ.45.3.3

Smith, L. B. (2000). How to learn words: An associative crane. In R. Golinkoff, \& K. Hirsh-Pasek (Eds.), Breaking the word learning barrier (pp. 51-80). Oxford: Oxford University Press. doi:10.1093/acprof:oso/9780195130324.003.003

Sweller, J., van Merrienboer, J., \& Paas, F. (1998). Cognitive architecture and instructional design. Educational Psychology Review, 10, 251-296. doi:10.1023/A:1022193728205

Whitehurst, G. J. (US Department of Education) (2008). Rigor and relevance redux: Director's biennial report to congress (IES 20096010). http://www.eric.ed.gov/PDFS/ED503384.pdf

Vedeler, L. (1997). Dramatic play: A format for "literate” language? Journal of Educational Psychology, 2, 153-167.

Verhallen, M. J. A., \& Bus, A. G. (2012). Beneficial effects of illustrations in picture storybooks for storing and retaining story text. Paper presented at the annual meeting of the Society for the Scientific Studies of Reading, Montreal.

Verhallen, M. J. A. J., Bus, A., \& de Jong, M. T. (2006). The promise of multimedia stories for kindergarten children at risk. Journal of Educational Psychology, 98, 410-419. doi:10.1037/0022-0663.98.2.410

Wittrock, M. C. (1981). Reading comprehension. In F. J. Pirozzolo, \& M. C. Wittrock (Eds.), Neuropsychological and cognitive processes in reading (pp. 229-259). New York: Academic-Press.

Wright, T. (2012). What classroom observations reveal about oral vocabulary instruction in kindergarten. Reading Research Quarterly, 47, 353-355. 


\section{Appendix}

Example of say-tell-do vocabulary instruction.

\section{BEFORE READING}

[Following introducing title, author, illustrator]

T: We're going to learn some new words in our story today The Little Red Hen. Let's talk about them together.

$\mathrm{T}$ (pointing to a photo): This is wheat. I say wheat; Now you say...

C: Wheat.

T: Right $\cdots$ wheat.

T: Let me tell you about wheat. Wheat is a grain that we use to make bread. Wheat is a grain we also have in our cereal. Can you turn and tell your friend this about wheat?

C: (turning to a friend) Wheat is in our cereal.

T: Yes $\cdots$ wheat is a grain. It is tall and sways in the wind, like this (moves hands back and forth). Can you do like I do? (Repeats motion,)

C: (hands move back and forth).

[T continues in same way with a set of new words.]

\section{DURING READING}

$\mathrm{T}$ : (pointing to the text illustration). Here it is! This is the wheat. You say:
C: Wheat.

T: Remember $\cdots$ wheat is a grain to make bread. It's tall and golden. Can you turn and tell your friend about wheat?

C: (to a friend). Wheat is tall. It's a grain.

$\mathrm{T}$ : Wheat sways in the wind, like this (moves arms back and forth). Do like I do to show wheat swaying in the wind.

C: (Move hands back and forth).

[As needed $\mathrm{T}$ repeats say-tell-do with other new words during reading.]

\section{AFTER READING}

[Following discussion about favorite parts of the story].

T: Let's use these puppets to retell the story of The Little Red Hen.

T: (pointing to photo or text illustration). We learned about wheat. You say:

C: Wheat.

$\mathrm{T}$ : Wheat is a grain we use to make bread. It's a grain in our cereal, too. It is tall and sways in the wind. Maybe you have seen wheat in a field. Turn and tell about wheat to your friend.

C: Wheat is a grain, and it's in bread.

T: Right! Can you show me how wheat sways in the wind?

C: (Move hands back and forth).

[T repeats other words as needed or as time permits.] 